sex/age-matched healthy controls: 50 in Cohort 1, 50 in Cohort 2, and 100 in Cohort 3. Among these 446 subjects, $75 \%$ were females and the average age was 52.5 years. Amine/phenol-containing metabolites were labeled by ${ }^{12} \mathrm{C}$-dansyl chloride to improve the LC-MS detection. For each cohort, a pooled sample was prepared and labeled by ${ }^{13} \mathrm{C}$-dansyl group to serve as the reference sample for relative quantification. Then the individual samples and the pooled sample were mixed 1:1. Finally, an LC-QTOF-MS platform analyzed the mixtures and output the intensity ratios of ${ }^{12} \mathrm{C} /{ }^{13} \mathrm{C}$ peak pairs.

Results: 1,149 amine/phenol-containing metabolites were commonly detected across the three sample sets. Among them, 134 were positively identified by our dansyl-labeling standard library, and 141 were matched to predicted retention times and mass values of dansyl-labeled human metabolites. Visualized by the partial least squares discriminant analysis (PLS-DA), the overall amine/ phenol-submetabolome demonstrated clear and consistent differences between healthy controls and the RA groups, with cross-validation $\mathrm{Q} 2=0.765,0.745$, 0.793 , respectively. The selection of significant metabolites was conducted according to the fold change and false-discovery-rate-adjusted Welch's t-test. Cohort 1 demonstrated 85 metabolites having higher concentrations in the RA samples than the controls, and 89 metabolites with lowered concentrations. The numbers of increased/decreased metabolites in Cohort 2 and 3 were $87 / 26$ and $90 / 53$, respectively. Importantly, there were 59 significantly discriminatory metabolites commonly found in the three data sets ( 49 increased and 9 decreased). We picked the top three with the highest univariate classification performance to form a biomarker panel. We implemented the linear support vector machine (SVM) to build the classifier and the receiver operating characteristic (ROC) analysis to measure the performance. The area-under-the-curve (AUC) values (95\% confidence interval) were 1.000 (1.000-1.000), 0.992 (0.967-1.000) and 0.902 (0.858$0.945)$ for the three cohorts, respectively. The results revealed the importance of examining multiple sample sets and even in the worst case (Cohort 3), our biomarker candidates could differentiate RA at $82.5 \%$ sensitivity and $82.5 \%$ specificity. Particularly, in Cohort 3, there were 30 RA patients negative for anti-cyclic citrullinated peptide and rheumatoid factor, and our metabolite panel demonstrated consistently high performance for differentiating these specific subjects from healthy controls.

Conclusion: Metabolites showing significant and consistent changes associated with RA have been identified with high discriminative power.

Disclosure of Interests: Wei Han: None declared, Xiaohang Wang: None declared, Liang Li: None declared, Stephanie Wichuk: None declared, Edna Hutchings: None declared, Rana Dadashova: None declared, Joel Paschke: None declared, Walter P Maksymowych Grant/research support from: Received research and/or educational grants from Abbvie, Novartis, Pfizer, UCB, Consultant of: WPM is Chief Medical Officer of CARE Arthritis Limited, has received consultant/participated in advisory boards for Abbvie, Boehringer, Celgene, Eli-Lilly, Galapagos, Gilead, Janssen, Novartis, Pfizer, UCB, Speakers bureau: Received speaker fees from Abbvie, Janssen, Novartis, Pfizer, UCB.

DOI: 10.1136/annrheumdis-2020-eular.5189

\section{THU0106 PREDICTORS OF FLARE IN RHEUMATOID ARTHRITIS PATIENTS WITH LOW DISEASE ACTIVITY}

K. W. Moon ${ }^{1} .{ }^{1}$ Kangwon National University Hospital, Internal Medicine, Chuncheon, Korea, Rep. of (South Korea)

Background: Low disease activity (LDA) in patients with rheumatoid arthritis (RA) are usually recognized as stable state. In according to most guidelines for RA, monotherapy of disease modifying anti-rheumatic drug (DAMRD) was recommended for RA patents with LDA. But some of patients with LDA suffer from flare in their disease course. Until now, we don't have enough data on factors that can predict flare in RA patients with LDA.

Objectives: The aim of this study is to evaluate predictor of flare in RA patient with LDA from long-term (3 year) cohort data.

Methods: Korean observational study network for arthritis (KORONA) registry is a nationwide Korean RA specific cohort registry that collecting data annually from 5,376 RA patients in 23 centers across South Korea. We include the data from 1, 801 RA patients with LDA (28 -joint disease activity score (DAS 28) $<3.2$ at enrollment) who had consecutive data of DAS28 for 3 years. Flare was defined as an increase in DAS28 compared with baseline of $>1.2$ or $>0.6$ if concurrent DAS28 $\geq 3.2$. Cox regression analysis was used to identify baseline predictors of flare.

Results: Among 1,801 RA patients, 673 patients (37.4\%) experienced flare in 3 years. When we compare the baseline characteristics of both flare and nonflare group, more women and more non-adherent patients for medication were observed in flare group. Flare group had longer disease duration, lower EuroQol 5D score, higher health assessment questionnaire (HAQ) score, and higher erythrocyte sedimentation rate (ESR) than non-flare group at baseline. In multivariate analysis, physician's VAS, HAQ score, ESR, and poor adherence for medication were significant predictors of flare (Table 1).

Table 1. Multivariate analysis of prediction of flare with baseline variables

\begin{tabular}{lccc}
\hline Measure & Hazard ratio & 95\% Confidence Interval & P-value \\
\hline Female & 1.130 & $0.906-1.409$ & 0.280 \\
Age & 0.996 & $0.988-1.005$ & 0.414 \\
Physician's VAS & 1.008 & $1.002-1.013$ & $<0.01$ \\
Pain VAS & 1.002 & $0.998-1.006$ & 0.34 \\
EQ5D & 0.952 & $0.534-1.696$ & 0.87 \\
HAQ & 1.407 & $1.109-1.786$ & $<0.01$ \\
ESR & 1.008 & $1.002-1.014$ & $<0.01$ \\
Poor adherence & 1.272 & $1.047-1.545$ & $<0.05$ \\
\hline
\end{tabular}

VAS: Visual Analogue Scale; EQ5D: EuroQol 5D; HAQ: Health Assessment Questionnaire ESR: Erythrocyte Sedimentation Rate

Conclusion: RA patient who have risk factors for flare, even though their disease activity was low, require more proactive treatment.

References:

[1] Bechman K, Tweehuysen L, Garrood T, Scott DL, Cope AP, Galloway JB, et al. Flares in Rheumatoid Arthritis Patients with Low Disease Activity: Predictability and Association with Worse Clinical Outcomes. J Rheumatol. 2018;45(11):1515-21.

[2] Singh JA, Saag KG, Bridges SL, Jr., Akl EA, Bannuru RR, Sullivan MC, et al. 2015 American College of Rheumatology Guideline for the Treatment of Rheumatoid Arthritis. Arthritis Rheumatol. 2016;68(1):1-26.

[3] Sung YK, Cho SK, Choi CB, Park SY, Shim J, Ahn JK, et al. Korean Observational Study Network for Arthritis (KORONA): establishment of a prospective multicenter cohort for rheumatoid arthritis in South Korea. Semin Arthritis Rheum. 2012;41(6):745-51.

Disclosure of Interests: None declared

DOI: 10.1136/annrheumdis-2020-eular.5202

\begin{tabular}{|l|l}
\hline THU0107 & OBESITY PREDICTS RESPONSE TO NOT ALL \\
& BUT CERTAIN BIOLOGICAL / TARGETED DISEASE \\
& MODIFYING ANTI-RHEUMATIC DRUGS FOR \\
& RHEUMATOID ARTHRITIS - RESULTS FROM KANSAI \\
& CONSORTIUM FOR WELL-BEING OF RHEUMATIC \\
& DISEASE PATIENTS (ANSWER COHORT)
\end{tabular}

K. Murakami ${ }^{1}$, M. Hashimoto ${ }^{2}$, K. Murata $^{2}$, W. Yamamoto ${ }^{2,3}$, R. Hara ${ }^{4}$, M. Katayama ${ }^{5}$, A. Onishi ${ }^{6}$, K. Akashi ${ }^{6}$, K. Nagai ${ }^{7}$, Y. Son ${ }^{8}$, H. Amuro ${ }^{8}$, T. Hirano ${ }^{9}$, K. Ebina ${ }^{10}$, K. Nishitani ${ }^{2}$, M. Tanaka ${ }^{2}$, H. Ito ${ }^{11}$, K. Ohmura ${ }^{1}{ }^{1}{ }^{1}$ Kyoto University Graduate School of Medicine, Department of Rheumatology and Clinical Immunology, Kyoto, Japan; ${ }^{2}$ Kyoto University Graduate School of Medicine, Department of Advanced Medicine for Rheumatic Diseases, Kyoto, Japan; ${ }^{3}$ Kurashiki Sweet Hospital, Health Information Management, Kurashiki, Japan; ${ }^{4}$ Nara Medical University, the Center for Rheumatic Diseases, Kashihara, Japan; ${ }^{5}$ Osaka Red Cross Hospital, Department of Rheumatology, Osaka, Japan; ${ }^{6}$ Kobe University Graduate School of Medicine, Department of Rheumatology and Clinical Immunology, Kobe, Japan; ${ }^{7}$ Osaka Medical College, Department of Internal Medicine (IV), Takatsuki, Japan; ${ }^{8}$ Kansai Medical University, First Department of Internal Medicine, Hirakata, Japan; ${ }^{9}$ Osaka University Graduate School of Medicine, Department of Respiratory Medicine and Clinical Immunology, Suita, Japan; ${ }^{10}$ Osaka University Graduate School of Medicine, Department of Musculoskeletal Regenerative Medicine, Suita, Japan; ${ }^{11}$ Kyoto University Graduate School of Medicine, Department of Orthopeadic Surgery, Kyoto, Japan

Background: A number of previous reports suggested that obesity is one of the baseline factors indicates refractory to biologic disease-modifying antirheumatic drugs (bDMARDs). However, difference of the significant responses appears on obesity patients depending on each kind of drug is yet unclear. However, it is yet unclear how the significant responses on obesity patients vary on each kind of drug.

Objectives: To assess whether obesity affects clinical outcome in rheumatoid arthritis (RA) treated with each molecular-targeted agent including bDMARDs and tofacitinib.

Methods: In Kansai consortium for well-being of rheumatic disease patients (ANSWER) cohort, which was the real-world retrospective cohort of clinical database for rheumatic diseases, RA patients who initiated biological / targeted disease modifying anti-rheumatic drugs were included and consecutively followed. Obesity was defined as BMI over than 25 , and patients were divided between 